\title{
Rash in COVID-19
}

\section{Shravan Kooragayalu}

Orange Regional Medical Center, Middletown, NY

Corresponding Author: Shravan Kooragayalu, Orange Regional Medical Center, 707, E Main Street, Middletown, NY

Received Date: July 29, 2020; Accepted Date: August 16, 2020; Published Date: September $21,2020$.

Citation: Kooragayalu S, (2020) Rash in COVID-19. J Clinical Case Reports and Studies, 1(6): Doi: 10.31579/2690-8808/018

Copyright: () 2020. Shravan Kooragayalu. This is an open-access article distributed under the terms of the Creative Commons Attribution License, which permits unrestricted use, distribution, and reproduction in any medium, provided the original author and source are credited.

\section{Abstract}

In December 2019, unexplained cases of pneumonia were reported in Wuhan, China. A novel coronavirus named Severe Acute Respiratory Syndrome coronavirus-2 (SARS CoV-2) was isolated from respiratory tract of patients, and the resultant disease was termed as COVID-19 (Coronavirus Disease 2019) (Jin YH 2020). COVID-19 has spread throughout china and across the world and it was declared as a pandemic by March $11^{\text {th }} 2020$ (Ng OT 2020).

Running Title: COVID-19 is a systemic disease that could affect almost all parts of the body but it primarily affects the respiratory system. There is very limited data in the literature about the dermatological manifestations of COVID19. An interesting case of skin rash in a patient who was critically ill with COVID-19 is presented here. The skin rash improved after short course of treatment with steroids. It is recommended that clinicians have a high index of suspicion to COVID-19 disease in patients who develop unexplainable rash.

Keywords: COVID-19; pneumonia; Severe Acute Respiratory Syndrome coronavirus-2 (SARS CoV-2)

\section{Introduction:}

In December 2019, unexplained cases of pneumonia were reported in Wuhan, China. A novel coronavirus named Severe Acute Respiratory Syndrome coronavirus-2 (SARS CoV-2) was isolated from respiratory tract of patients, and the resultant disease was termed as COVID-19 (Coronavirus Disease 2019) (Jin YH 2020). COVID-19 has spread throughout china and across the world and it was declared as a pandemic by March $11^{\text {th }} 2020$ (Ng OT 2020).

COVID-19 is a systemic disease that could affect almost any organ of the body but it primarily affects the respiratory system. The known underlying mechanism so far is from systemic cytokine release. COVID19 is mainly suspected by clinical symptoms of fever, fatigue, dry cough, anorexia, dyspnea, rhinorrhea, aguesia, anosmia and measurement of vital parameters like temperature, pulse oximetry saturation and on radiological images including Chest $\mathrm{X}$ ray, Chest Computed Tomography scans (Wang D 2020). Laboratory findings could often demonstrate lymphopenia and elevated Lactate Dehydrogenase. The diagnosis is confirmed by isolation of the virus from nasopharyngeal and/or oropharyngeal swab.

So far, there is very limited data in the literature about the dermatological manifestations in COVID-19. An interesting case of skin rash in a patient who was critically ill with COVID-19 is presented here.

\section{Case:}

A 55-year-old man presented to the hospital with difficulty breathing, cough and fevers for 3 days. His chest $\mathrm{x}$ ray revealed diffuse bilateral patchy opacities and a Computed Tomography of the chest with contrast confirmed the X-ray findings and also showed extensive bilateral pulmonary embolic disease. He developed worsening hypoxic respiratory failure and he underwent endotracheal intubation. He was started on broad spectrum antibiotics including vancomycin, cefepime and azithromycin. He was also started on Heparin drip. He was tested positive for COVID19. All the antibiotics were discontinued and patient was started on hydroxychloroquine for a total of 5 days. Over the course of the hospitalization he developed non-oliguric acute renal failure and needed 3 sessions of intermittent hemodialysis. On day 8 day of hospitalization patient developed a diffuse, erythematous and pustular rash which was blanching in nature and was located predominantly in the upper torso, abdomen, upper back and upper thighs. He did not have any rash on the palms, soles or mucous membranes. Significant laboratory findings including bilirubin, eosinophils, anti-nuclear antibody, platelets were within normal limits. A short course of prednisone $40 \mathrm{mg}$ daily for total 5 days was started. There was a dramatic improvement in the rash starting from day 10 (Figure 1). The overall clinical status of the patient improved, he was extubated and discharged home on day 17 after admission.

\section{Discussion:}

After detailed review there was no association or temporal relation of any of the medications administered that could have caused similar rash in this patient. Patient was on Vancomycin and Cefepime for the first 2 days when the results of the COVID-19 were pending.

There have been reports of COVID-19 initially presenting only as rash like symptom and misdiagnosed as another common disease (Beuy Joob 2020). In a report from Italy it is documented that almost $20 \%$ of COVID19 patients develop cutaneous manifestations. The rash could occur before or during hospitalization. In that study, trunk was the main affected part and the rash did not have any co-relation with the disease severity (RECALCATI 2020). 
Given the pandemic situation, biopsy of the skin rash was not performed which would have given more information regarding its pathophysiology. COVID-19 can cause different types of skin rash depending on the mechanism. The rash can be petechial or tiny bruises, and transient livedoid eruptions. It could feature signs of small blood vessel occlusion. Whether it is neurogenic, micro thrombotic, or immune complex mediated is still unknown.

\section{Conclusion:}

It is recommended that clinicians have a high index of suspicion to COVID-19 disease in patients who develop unexplainable rash. This is one of the first reports to show COVID-19 rash responding to steroids. Indisputably, we need more studies to confirm and better understand the dermatological manifestations of COVID-19.

\section{Abbreviations:}

SARS CoV-2: Severe Acute Respiratory Syndrome coronavirus-2

COVID-19: Coronavirus Disease 2019

\section{Acknowledgements:}

The author acknowledge that they have contributed to the material of the manuscripts, read and have approved.

\section{Conflict of Interest: None}

\section{Consent: Obtained}

\section{Funding Source: None}

Nothing to Disclose

\section{References}

1. Beuy Joob, Viroj Wiwanitkit. (2020). "COVID-19 can present with a rash and be mistaken for dengue." (Journal of American Academy of Dermatology).

2. Jin YH, Cai L, Cheng ZS et al. (2020). "A rapid advice guideline for the diagnosis and treatment of 2019 novel coronavirus (2019-nCoV) infected pneumonia (standard version)." (Mil Med Res) 7, 4

3. Ng OT, Marimuthu K, Chia PY, et al. (2020). "SARS-CoV-2 Infection among travelers returning from Wuhan, China." (New England Journal of Medicine)

4. RECALCATI, SEBASTIANO. "Cutaneous manifestations in COVID-19: a first perspective." March 2020.

5. Wang D, Hu B, Hu C et al. (2020). "Clinical Characteristics of 138 Hospitalized Patients With 2019 Novel CoronavirusInfected Pneumonia in Wuhan, China." (JAMA) 